\section{MS6-P8 PR-10: one fold for many phytohormones?}

Mariusz Jaskolski ${ }^{1,2}$, Milosz Ruszkowski ${ }^{1}$, Joanna Sliwiak ${ }^{1}$

1. Center for Biocrystallographic Research, Institute of Bioorganic Chemistry, Polish Academy of Sciences, Poznan, Poland

2. Department of Crystallography, Faculty of Chemistry, A. Mickiewicz University, Poznan, Poland

\section{email: mariuszj@amu.edu.pl}

Plant Pathogenesis-Related proteins of class 10 (PR-10) are abundant multigene proteins differentially expressed in various organs in stressful conditions, such as pathogen invasion. They have a canonical fold consisting of a seven-stranded antiparallel $\beta$-sheet gripped over a long C-terminal helix $\alpha 3$, whose free end rests on a V-shaped support formed by two shorter helices. A bizarre feature of the PR-10 fold is a huge hydrophobic cavity enclosed by helix $\alpha 3$ and the $\beta$-sheet. Amazingly, this internal void does not lead to instability, as in biochemical and mechanical studies the PR-10 proteins appear to be even more stable and robust than typical globular proteins. In a number of crystallographic and biophysical studies, the PR-10 proteins have been shown to bind plant hormones from entirely disjointed chemical groups. So far, proteins with PR-10 fold have been studied in complexes with cytokinins, brassinosteroids, gibberellins, abscisic acid and melatonin. The emerging picture is, however, extremely puzzling because not only can the same PR-10 protein bind phytohormones from entirely different groups, but the same ligands are often found in different binding modes and with variable stoichiometry. These perplexing observations seem to contradict our notion of specificity of protein-ligand recognition but may hold the key to the puzzle of the biological function of PR-10 proteins.

Keywords: pathogenesis-related proteins, phytohormones, plant signal transduction

\section{MS6-P9 Structural basis for protein translocation and insertion in the outer membrane by Omp 85 proteins}

Fabian Gruss ${ }^{1}$, Roman P. Jakob ${ }^{1}$, Bernard Clantin ${ }^{2,3}$, Vincent Villeret $^{2,3}$, Sebastian Hiller ${ }^{1}$, Timm Maier ${ }^{1}$

1. Biozentrum, University of Basel, Switzerland

2. CNRS UMR8576, Unité Glycobiologie Structurale et Fonctionnelle, Villeneuve d'Ascq, France

3. Univ. Lille Nord de France, Lille, France

email: fabian.gruss@unibas.ch

Omp85 proteins transport protein substrates across or insert them into the outer membrane of Gram-negative bacteria. Their general architecture comprises a C-terminal 16 -stranded transmembrane $\beta$-barrel pore and one or more periplasmic N-terminal POTRA domains, which mediate initial substrate interactions.

We determined the crystal structure of the Omp85 insertase TamA, which is involved in autotransporter biogenesis in E. coli (1). The TamA barrel shows reduced interactions between the first and last strand and a lipid-occupied lateral gate, which together suggest hybrid-barrel formation and lateral release as a plausible membrane insertion mechanism. Two highly conserved signature motifs in Omp85 proteins, located in the extracellular L6 loop and the inner barrel wall, respectively, interact and stabilize the closed barrel. Equivalent features were also observed in the crystal structure of the Omp85 protein BamA, the general outer membrane insertase (2). The requirement for lateral barrel opening between strands 1 and 16 was confirmed by crosslinking (3). Contrasting observations were made for the Omp85 translocase FhaC from $B$. pertussis, which secretes filamentous hemagglutinin to the extracellular space and is involved in bacterial virulence. Here, a crystal structure visualized loop L6 in a different conformation, without interaction of the two signature motifs (4).

To analyze the differences between Omp85 translocases and insertases, we determined the crystal structure of an FhaC variant defective in substrate recognition. In this structure the L6 loop conformation is equivalent to the one of TamA and BamA (5). Re-analysis of the original wt-FhaC crystal structure revealed that the L6 loop was affected by mismodeling and adopts identical conformations in all Omp85 structures. This conformation is thus a structural characteristic of the entire Omp85 family and provides a common structural basis for both protein translocation and insertion. Furthermore, the structure of the FhaC variant reveals a mechanism for substrate selection based on FhaC plug-helix release and linker competition, which may well be relevant for other Omp85 translocases involved in two-partner secretion.

1) Gruss F. et al., Nat. Struct. Mol. Biol. 20, 1318-1320 (2013).

2) Noinaj N. et al., Nature 501, 385-390 (2013).

3) Noinaj N. et al., Structure 22, 1055-1062 (2014).

4) Clantin B. et al., Science 317, 957-961 (2007).

5) Maier T. et al., Nat. Commun. 6, 10.1038/Ncomms8452 (2015).

Keywords: Omp85, TamA, FhaC, TPS, two-partner secretion, autotransporter, outer membrane 